\title{
Citrus Peel Ethanol Extract Inhibits the Adipogenesis Caused from High Fat-Induced DIO Model
}

\author{
Mustafa Zafer Karagozlu1, Minjin Kim², Myoungsook Lee ${ }^{1,2^{*}}$ \\ ${ }^{1}$ Research Institute of Obesity Sciences, Sungshin Women's University, Seoul, Republic of Korea \\ ${ }^{2}$ Department of Food and Nutrition, Sungshin Women's University, Seoul, Republic of Korea \\ Email: *mlee@sungshin.ac.kr
}

Received 1 December 2015; accepted 17 January 2016; published 20 January 2016

Copyright @ 2016 by authors and Scientific Research Publishing Inc.

This work is licensed under the Creative Commons Attribution International License (CC BY). http://creativecommons.org/licenses/by/4.0/

c) (7) Open Access

\section{Abstract}

Background: Flavonoids are multi-functional bioactive compounds that have been used as natural compounds against various diseases. Citrus fruit is an important source for bioactive flavonoids with potential anti-obesity benefits. Methods: To determine the anti-obese effects of citrus peel, a 45\% high fat diet-induced obesity (DIO) model using C57BL/6 mice was prepared for 10 weeks and then treated orally for 12 weeks with ethanol extracts of citrus peel $(300 \mathrm{mg} / \mathrm{kg}, \mathrm{CP})$. CP was compared with normal chow diet (C), high fat diet (HF), and the anti-obesity drug orlistat (30 $\mathrm{mg} / \mathrm{kg}, 0$ ) as a positive control. HF caused increases in lipid accumulation, body weight gain, and hepatic toxicity compared with the $\mathrm{C}$ group. Results: CP treatment reduced body weight gain and decreased epididymal fat, mesenteric fat, and plasma and hepatic TG levels in a similar manner as 0 treatment. Besides, CP was comparatively more effective than 0 at increasing high density lipoprotein cholesterol (HDL-c) while reducing hepatic toxicity, which is caused by HF. Fat accumulation in adipose tissue was decreased by CP treatment because of up-regulation of specific lipolysis enzymes such as HSL and AMPK and down-regulation of adipogenesis related genes such as C/EBP $\alpha$ and ACC. The proinflammatory cytokines, TNF- $\alpha$ and IL-6, which are the key factors for regulation of inflammation, were significantly decreased by $\mathrm{CP}$. Conclusion: $\mathrm{CP}$ may be a potential natural source for new anti-obesity candidate because of its inhibitory effect on fat synthesisrelated inflammation and its positive effect on lipolysis activation.

\section{Keywords}

Citrus unshiu, Obesity, Inflammation, Hesperidin, Naruritin

\footnotetext{
${ }^{*}$ Corresponding author.
} 


\section{Introduction}

Obesity is observed to be in a close relationship with low-grade systemic inflammation, which is considered to have a tendency towards the progression of insulin resistance, hyperglycemia, and related metabolic disorders [1] [2]. Moreover, from evidence over the past decades, adipose tissue has been placed in a pivotal position for the regulation of lipid and glucose metabolism. Adipocytes are shown to secrete numerous bioactive molecules, principally proteins such as anti- and pro-inflammatory adipokines, which play crucial roles in inflammatory responses [3] [4]. It has been suggested that in obesity the dysfunction of adipocytes and macrophages along with the deteriorated secretion of adipokines results in chronic inflammatory response [3]. Elevated levels of pro-inflammatory adipokines such as interleukin-6 (IL-6) and tumor necrosis factor-alpha (TNF- $\alpha$ ) are mainly associated with increasing adiposity and malfunctioning of adipocytes in obesity [5]. It has been speculated that obesity-linked inflammation and cell damage are closely linked through adipocyte lipolysis. In the TNF-alpha signaling pathway, AMPK is an important target and TNF- $\alpha$ has a suppressive effect on the AMPK signaling pathway, which regulates lipolysis and fat oxidation in adipocytes [6].

Citrus fruits are primarily used in juice and related industries, and the production method leaves significant amounts of by-products such as peel, pulp, and seeds. These by-products have been utilized in several processes including production of stock feed, oils, and food flavor [7]. However, recent studies credit the citrus byproducts with containing large amounts of flavonoids and polyphenols that have health benefits [8] [9]. Citrus unshiu is a commonly consumed fruit throughout countries such as Korea, Japan, and China. In recent years, studies have explored the presence of some potentially bioactive compounds in $C$. unshiu and its inedible byproducts such as peels and seeds. It has been reported that peel extracts contain large amounts of flavonoids, such as hesperidin and narirutin [7]. In addition, C. unshiu peel extracts were shown to possess significant health-beneficial properties such as antioxidant, anti-inflammatory, anti-cancer, and anti-obesity [10]-[13]. Considering the potential held by $C$. unshiu in terms of health benefits and the urge to control and treat obesitylinked inflammation, this study focused on the effect of $C$. unshiu peel extract on relieving high-fat diet-induced adiposity-linked inflammation and suggested a possible pathway of the anti-inflammatory mechanism in obesity induced mice.

\section{Experimental}

\subsection{Preparation of Ethanol Extract of Citrus Peel (CP)}

Fresh C. unshiu fruits were purchased in November 2012 from The Village Green (Jeju, South Korea) and peeled. The $C$. unshiu peel was dried at $56^{\circ} \mathrm{C}$ for $8 \mathrm{~h}$, and then $7 \mathrm{~L}$ of $70 \%$ ethanol (Daehan Ethanol Life Co., Seoul, South Korea) was added to $1.823 \mathrm{~kg}$ of sample. After $3 \mathrm{~h}$ extraction at $75^{\circ} \mathrm{C}$, the sample was cooled, filtered, and evaporated (EYELA N1100, Tokyo Rikakikai Co., Tokyo, Japan). The final concentrate collected was a 445.7 g extract with 16.13\% moisture content. Next, high performance liquid chromatography (HPLC, HP 1100, Agilent Technologies, California, USA) analysis was performed on the sample to detect its flavonoid composition. The CP sample was dissolved in methanol and injected into a $5 \mu \mathrm{m}$ Supercosil LC-18 column. (250 × 4.6 mm Sigma-Aldrich Corp., St. Louis, USA). Hesperidin (Sigma-Aldrich Corp., St. Louis, USA) dissolved in $0.1 \mathrm{M} \mathrm{NaOH}$ and narirutin (Exstrasynthese, Lyon, France) dissolved in water were used as standards. Acetonitrile and $0.5 \%$ acetic acid were used as mobile phase solvents in a two solvent pumping HPLC system. The samples were injected at time 0 and the gradient profile started at $0 \%$ for solvent mixture A and was changed to $85 \%$ A by $35 \mathrm{~min}$. The flow-rate was adjusted at $1.0 \mathrm{~mL} / \mathrm{min}$ and the column temperature was maintained at $30^{\circ} \mathrm{C}$ during all runs.

\subsection{Animal Experiment}

All animal studies were carried out in accordance with the ethical guidelines of the Experimental Animal Holding of Sungshin Women's University (Approval number: SSWU AEC 2011-001). Twenty four 4-week old male mice were purchased from Orient Bio (Seoul, South Korea). After a 1 week adaptation period, mice were divided randomly into two groups. The control group $(C, n=6)$ were fed with chow diet (D12450B, Research Diet Inc, NJ, USA), while the other group $(n=18)$ were fed with $45 \%$ high fat diet for 10 weeks to induce DIO mice. For 12 week experimental periods, the DIO mice were distributed randomly into three groups: only high fat group (HF), citrus peel extracts group (CP) fed $300 \mathrm{mg} / \mathrm{kg}$ CP with high fat diet, and orlistat group (O) fed 
with $30 \mathrm{mg} / \mathrm{kg}$ orlistat with high fat diets. Ingredients of all the experimental diets are shown in Table 1. During the experimental procedures, all animals were kept at a controlled temperature $\left(22^{\circ} \mathrm{C} \pm 2^{\circ} \mathrm{C}\right)$ in a $12 \mathrm{~h}$ light-dark cycle room with free access to food and distilled water. Food intake was measured twice a week and body weight was measured once a week. At the end of the experimental period, $12 \mathrm{~h}$ starved mice were anesthetized by diethyl ether and blood samples were taken from heart. Furthermore, liver and adipose tissue (mesentic fat, epididymal fat, and subcutaneous fat) were collected and stored at $-80^{\circ} \mathrm{C}$ until further assay.

\subsection{Plasma Biochemistry}

Total cholesterol (TC), triglycerides (TG), and high density lipoprotein cholesterol (HDL-c) in blood plasma were analyzed by an enzymatic colorimetric method using assay kits (Human 10028, 10724, 10084, Wiesbaden, Germany). Likewise the TG and TC concentrations of hepatic tissues were also analyzed with the same method after extraction of lipid in the tissues by the Folch method [14]. Low density lipoprotein cholesterol (LDL-c) was calculated by the Friedewald formula and the atherogenic index (AI) was calculated by the Rosenfeld formula [15]. Aspartate aminotransferase (AST) and alanine aminotransferase (ALT) were measured with assay kit (Bio Vision K753-100, K752-100, CA, USA) to detect tissue toxicity, and the total value calculated by measuring optical absorbance using a microplate reader (Thermo, MA, USA).

\subsection{Adipocyte Morphology}

Morphological changes of epididymal adipose tissue were examined. Briefly, collected epididymal adipose tissue was fixed in $12 \%$ neutral formalin solution. After fixation for $12 \mathrm{~h}$, the samples were dehydrated with ethanol and washed with xylene for embedding in paraffin. Finally, $0.4 \mu \mathrm{m}$ sections of the samples were prepared for hematoxylin-eosin staining (H\&E, Sigma-Aldrich Corp., St. Louis, USA). Any morphological changes of the stained samples were determined microscopically by using an inverted microscope (Nikon Eclipse, Tokyo, Japan) and calculated by using an image analysis software (ImageJ, NIH, MD, USA).

\subsection{Western Blotting}

The collected mesenteric tissue of mice was lysed in ice-cold RIPA buffer for extraction and the protein amount

Table 1. Compositions of experimental diets in high fat diet induced DIO model.

\begin{tabular}{ccccc}
\hline & Chow Diet & 45\% high fat diet-induced model & O \\
\cline { 2 - 5 } & C & HF & CP & 800 \\
Casein & 800 & 800 & 800 & 12 \\
L-Cysteine & 12 & 12 & 12 & 291 \\
Corn Starch & 1260 & 291 & 291 & 400 \\
Maltodextrin 10 & 140 & 400 & 400 & 691 \\
Sucrose & 1400 & 691 & 691 & 225 \\
Soybean Oil & 225 & 225 & 225 & 40 \\
Lard & 180 & 1598 & 1598 & - \\
Vitamin Mix: V10001 & 40 & 40 & 40 & 30 \\
Citrus Peel (mg/kg) & - & - & 300 & - \\
Orlistat (mg/kg) & - & - & 20 & 35 \\
Protein & 20 & Percentage of total energy (\%) & 35 & 45 \\
Carbohydrate & 70 & 20 & 45 & \\
Fat & 10 & 35 & & \\
\hline
\end{tabular}

C: Chow diet, HF: High fat diet, CP: High fat diet + Citrus peel extract (300 mg/kg), O: High fat diet + Orlistat (30 mg/kg). 
collected was measured by the Bradford method. Equal amounts of protein were separated by sodium dodecyl sulfate-polyacrylamide gel electrophoresis (SDS-PAGE), transferred onto a nitrocellulose membrane (ELPIS, Daejon, South Korea), and blocked in 5\% (w/v) skim milk. The membrane was then incubated for $2 \mathrm{~h}$ at room temperature with diluted 1:2000 primary antibody of monocyte chemoattractant protein-1 (MCP-1), TNF- $\alpha$, FAS, phosphorylated AMP-activated protein kinase (P-AMPK), AMP-activated protein kinase (AMPK), phosphorylated hormone sensitive lipase (P-HSL), hormone sensitive lipase (HSL), and $\alpha$-tubulin. Membrane-bound labeled protein bands were visualized by ECL western blotting solution and detected by chemiluminescence with X-ray films. Finally, the band densities of western blot were determined using an image analysis software (ImageJ, NIH, MD, USA).

\subsection{RT-PCR}

Mesenteric adipose tissue was homogenized by using TRIzol reagent (Invitrogen, MA, USA) according to the manufacturer's instructions. Briefly, total RNA $(1 \mu \mathrm{g})$ and $1 \mu \mathrm{l}$ oligo (dT) were preheated and reverse transcribed in a master mix containing $5 \times$ RT reaction buffer, $8 \mathrm{mM}$ DTT, Diastar RTase, RNase-free water, and RNA inhibitor at $50^{\circ} \mathrm{C}$ for $60 \mathrm{~min}$ and at $95^{\circ} \mathrm{C}$ for $5 \mathrm{~min}$ using an automatic thermocycler (iCycleriQ BIO-RAD, CA, USA). The target cDNA was amplified using the following primers: forward 5'-ACA-GGG-AGG-AAGCAA-TCT-CCC-TGC-3' and reverse 5'-ACT-GCC-GGG-TCA-CCT-TAA-GTA-3' for acetyl-CoA carboxylase (ACC) gene; forward 5'-CTT-CAC-AAG-TCC-GGA-GAG-GAG-3' and reverse 5'-TGG-TCT-TGG-TCC-TTAGCC-ACT-3' for (IL-6) gene; forward 5'-AAC-TAT-GAG-GTG-GAT-CGG-AG-3' and reverse 5'-TCC-ATACCG-CAT-TAC-CAT-GC-3' for AMPK gene; forward 5'-GAT-GAG-AAG-TTC-CCA-ATG-GC-3' and reverse 5'-CGG-ACT-CCG-CAA-AGT-CTA-AGT-3' for TNF- $\alpha$ gene; forward 5'-CCT-CTT-CAA-CAG-CAA-CCACAA-3' and reverse 5'-ACA-ACT-CCA-CCA-AGC-TTCT-GCT-3' for CCAAT/enhancer binding protein alpha (C/EBP- $\alpha$ ) gene; and forward 5'-CTG-AAG-GGC-ATC-TTG-GGC-3' and reverse 5'-TTA-CTC-CTT-GGAGGC-CAT-G-3' for glyceraldehyde-3-phosphate dehydrogenase (GAPDH) gene. The amplification was carried out at $94^{\circ} \mathrm{C}$ for $5 \mathrm{~min}, 55^{\circ} \mathrm{C}$ for $30 \mathrm{sec}$, and $70^{\circ} \mathrm{C}$ for $45 \mathrm{sec}$. After 35 cycles, the PCR products were analyzed by electrophoresis on $2 \%$ agarose gel for $30 \mathrm{~min}$ at $120 \mathrm{~V}$. Gels were then stained with Redsafe Tm nucleic acid staining solution (iNtRON, Gyeonggi, Korea) and visualized by UV light.

\subsection{Statistical Analysis}

Data were expressed as mean \pm SD. Differences between the means of individual groups were assessed by one-way ANOVA with Duncan's multiple range tests. Significance of differences was expressed at the $\mathrm{p}<0.05$ level.

\section{Results}

\subsection{HPLC Results}

The flavonoid composition of CP was detected by HPLC analysis, and from the results it was observed that the major flavonoid content of the sample was composed of narirutin $(26.93 \mathrm{mg} / \mathrm{g})$ and hesperidin $(20.05 \mathrm{mg} / \mathrm{g})$. Narirutin accounted for $46.25 \%$ of total flavonoid content, while hesperidin accounted for $35.61 \%$ (Figure 1).

\subsection{Body Weight Gain and Food Efficiency Ratio}

Body weights and total fat of adipose tissues of all tested groups were measured and food efficiency ratios were calculated (Table 2). CP treatment decreased the body weight in high fat diet induced obese mice and orlistat promoted body weight loss after 12 weeks of treatment. Besides, there was a significant decrease in epididymal, mesenteric, and subcutaneous fat weight with CP and orlistat treatment (Figure 2). Calculation of the amounts of mesenteric, epididymal, and subcutaneous fat from adipose tissue in the $\mathrm{C}, \mathrm{HF}, \mathrm{CP}$, and $\mathrm{O}$ groups showed that the HF group had the highest and the $\mathrm{O}$ group had the lowest fat adipose tissue weights. A comparison of the HF and CP groups shows a dramatic decrease in mesenteric and epididymal fat weights with CP treatment, but there were no significant difference in subcutaneous fat weight between the CP and HF groups. Within the four groups, the HF and CP groups both showed body weight gain; however, there was a significantly higher gain in body weight for the HF group compared with the CP group. Also, the food efficiency ratio of the HF group was 


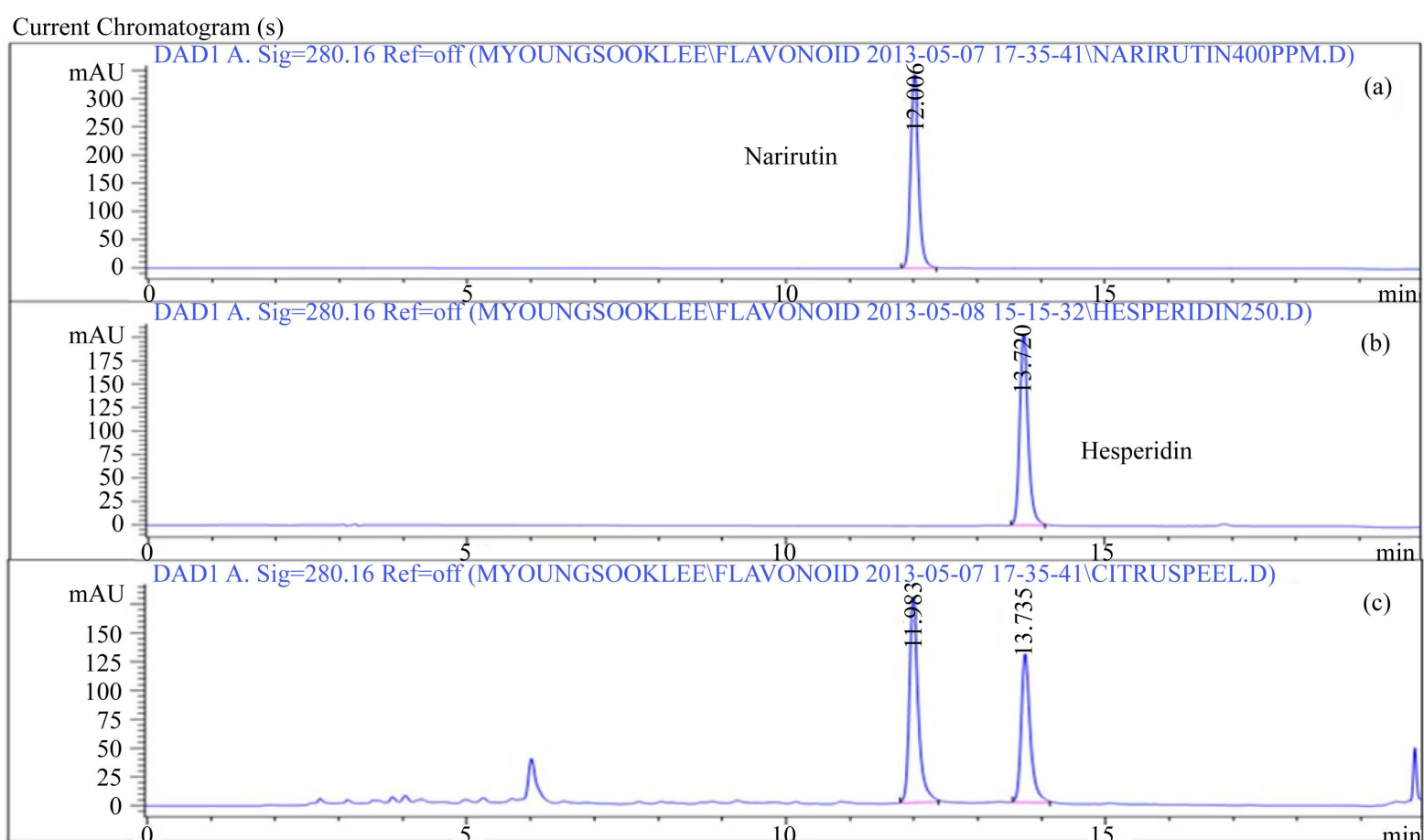

Figure 1. HPLC chromatogram for the standard of narirutin (a) and hesperidin (b), and both were detected in the CP extracts (c). Using HP 1100 (Agilent Technologies,USA), their condition was that column; LichroCART (LC-18, $5 \mu$ m, 250 × 4.6), detector; DAD $(530 \mathrm{~nm})$, inj. vol.; $20 \mu \mathrm{L}$, flow rate; $1 \mathrm{~mL} / \mathrm{min}$, solvent-I; water $(0.1 \% \mathrm{AcOH})$, solvent-II; AcCN $(0.5 \%$ $\mathrm{AcOH})$, and gradient condition was changed for 0 - 35 min.

notably higher than that of the CP group. The orlistat treatment group was the only group that exhibited a decrease in body weight.

\subsection{Blood Biochemistry and Hepatic Toxicity}

Measurements of TC and TG concentrations of hepatic tissue and blood plasma (Table 2) show that plasma and hepatic TG increased in the HF fed group. A comparison of the HF and CP groups shows a dramatic decrease in both hepatic and plasma TG with CP treatment. Especially plasma TG was two times higher in the HF group, while there was no significant difference for plasma TG levels between the CP and O groups (Figure 3). The effect of CP treatment on hepatic TG was also significant, but the effect was less than with orlistat treatment. In addition, the $\mathrm{O}$ group also exhibited the lowest total cholesterol concentration in both hepatic tissue and blood plasma; however, except for the $\mathrm{O}$ group, there was no statistically meaningful difference for total cholesterol concentration in both the hepatic tissue and blood plasma of the treated groups. In order to determine the lipid profile of blood plasma, HDL-c was measured and the composition of LDL-c and AI was calculated in accordance with the HDL-c amount (Table 2). After 12 weeks of treatment, all high fat diet-fed groups showed similar trends regarding lipid profile with decreased HDL-c levels coupled with significantly elevated LDL-c and AI. The HF group had the lowest HDL-c concentration and the highest LDL-c and AI values. In contrast, there was a significant decrease in LDL-c and significant increase in HDL-c with CP treatment. Among all groups, the CP group had the highest HDL-c and lowest LDL-c and AI. There were no significant differences between the CP and $\mathrm{O}$ groups for blood plasma LDL concentration. Furthermore, measurements of plasma AST and ALT showed that the C group had the lowest levels of AST and ALT while the HF group had highest levels of AST and ALT in blood plasma among all groups. Within the high fat-treated groups, there was no significant difference in regulation of plasma AST levels between the $\mathrm{O}$ and CP groups (Figure 3).

\subsection{Adipocytes Morphology in Epididymal Tissue}

From the morphological changes observed in epididymal tissue by H\&E staining and subsequent calculations 
Table 2. Citrus peel ethanol extracts changes body and fat mass, FER and blood and hepatic lipid profiles.

\begin{tabular}{|c|c|c|c|c|c|}
\hline \multirow{2}{*}{$\begin{array}{c}\text { Groups } \\
\text { Variables }\end{array}$} & \multirow{2}{*}{$\begin{array}{c}\text { Chow diet } \\
\text { C }\end{array}$} & \multicolumn{3}{|c|}{$45 \%$ high fat diet-induced model } & \multirow{2}{*}{ p-valu } \\
\hline & & HF & $\mathbf{C P}$ & $\mathbf{O}$ & \\
\hline \multicolumn{6}{|c|}{ BW (g) \& FER (\%) } \\
\hline Initial BW & $30.88 \pm 2.78^{\mathrm{a}}$ & $39.62 \pm 2.57^{\mathrm{b}}$ & $43.17 \pm 2.50^{c}$ & $40.56 \pm 3.11^{\mathrm{c}}$ & $<0.001$ \\
\hline Final BW & $32.08 \pm 2.90^{\mathrm{a}}$ & $45.48 \pm 1.71^{\mathrm{b}}$ & $47.59 \pm 2.97^{\mathrm{b}}$ & $30.72 \pm 2.88^{\mathrm{a}}$ & $<0.001$ \\
\hline BW gain & $1.20 \pm 0.82^{\mathrm{b}}$ & $5.86 \pm 1.57^{\mathrm{d}}$ & $4.42 \pm 0.71^{\mathrm{c}}$ & $-9.8 \pm 1.20^{\mathrm{a}}$ & $<0.001$ \\
\hline FER (\%) & $0.50 \pm 0.35^{\mathrm{b}}$ & $2.40 \pm 0.67^{\mathrm{c}}$ & $1.92 \pm 0.26^{\mathrm{c}}$ & $-3.47 \pm 0.53^{\mathrm{a}}$ & $<0.001$ \\
\hline \multicolumn{6}{|c|}{ Adipose tissue (g) } \\
\hline Epididymal & $4.46 \pm 0.96^{\mathrm{c}}$ & $5.65 \pm 0.50^{\mathrm{d}}$ & $3.57 \pm 0.58^{\mathrm{b}}$ & $2.73 \pm 0.46^{\mathrm{a}}$ & $<0.05$ \\
\hline Mesentric & $1.11 \pm 0.49^{\mathrm{b}}$ & $4.40 \pm 0.64^{\mathrm{d}}$ & $3.61 \pm 0.61^{\mathrm{c}}$ & $0.46 \pm 0.16^{\mathrm{a}}$ & $<0.001$ \\
\hline Subcutaneous & $2.37 \pm 0.38^{\mathrm{a}}$ & $6.55 \pm 0.81^{\mathrm{b}}$ & $5.49 \pm 1.25^{\mathrm{c}}$ & $2.05 \pm 0.84^{\mathrm{a}}$ & $<0.001$ \\
\hline Total fat & $7.68 \pm 1.87^{\mathrm{b}}$ & $12.68 \pm 1.22^{\mathrm{d}}$ & $12.68 \pm 0.53^{\mathrm{c}}$ & $4.29 \pm 0.96^{\mathrm{a}}$ & $<0.001$ \\
\hline \multicolumn{6}{|c|}{ Blood lipids (mg/dl) } \\
\hline TG & $93.10 \pm 17.98^{\mathrm{a}}$ & $152.62 \pm 4.12^{\mathrm{b}}$ & $83.57 \pm 14.29^{\mathrm{a}}$ & $62.29 \pm 18.90^{\mathrm{a}}$ & $<0.001$ \\
\hline TC & $407.29 \pm 31.46^{\mathrm{ab}}$ & $448.96 \pm 19.09^{\mathrm{b}}$ & $426.04 \pm 3.61^{\mathrm{b}}$ & $373.96 \pm 7.22^{\mathrm{a}}$ & $<0.05$ \\
\hline HDL-c & $48.47 \pm 0.57^{\mathrm{b}}$ & $45.90 \pm 0.29^{\mathrm{a}}$ & $50.75 \pm 0.57^{c}$ & $47.90 \pm 0.57^{\mathrm{b}}$ & $<0.001$ \\
\hline LDL-c & $340.21 \pm 35.30^{\mathrm{ab}}$ & $372.53 \pm 19.91^{\mathrm{b}}$ & $309.35 \pm 10.51^{\mathrm{a}}$ & $361.43 \pm 4.27^{\mathrm{b}}$ & $<0.05$ \\
\hline AI & $7.41 \pm 0.72^{\mathrm{b}}$ & $8.78 \pm 0.46^{\mathrm{c}}$ & $6.37 \pm 0.22^{\mathrm{a}}$ & $7.90 \pm 0.13^{b}$ & $<0.05$ \\
\hline \multicolumn{6}{|c|}{ Hepatic lipids (mg/dl/g protein) } \\
\hline TG & $18.65 \pm 2.07^{\mathrm{b}}$ & $22.03 \pm 1.42^{\mathrm{c}}$ & $15.63 \pm 1.47^{\mathrm{b}}$ & $7.71 \pm 2.05^{\mathrm{a}}$ & $<0.001$ \\
\hline TC & $2.03 \pm 0.06^{\mathrm{b}}$ & $1.95 \pm 0.02^{\mathrm{b}}$ & $1.91 \pm 0.07^{\mathrm{b}}$ & $1.73 \pm 0.09 a$ & $<0.05$ \\
\hline
\end{tabular}

C: Chow diet, HF: High fat diet, CP: High fat diet + Citrus peel $(300 \mathrm{mg} / \mathrm{kg})$, O: High fat diet + Orlistat $(30 \mathrm{mg} / \mathrm{kg})$. Mean \pm SE $(\mathrm{n}=6 \mathrm{for}$ each group). ${ }^{\mathrm{a}-\mathrm{d}}$ Means with the different letters are significantly different $(\mathrm{p}<0.05)$ by ANOVA and Duncan's multiple range test. Abbreviation: BW: body weight; FER: food efficiency rate; TG: triacylglycerol; TC: total cholesterol; LDL-c: LDL cholesterol; HDL-c: HDL cholesterol.
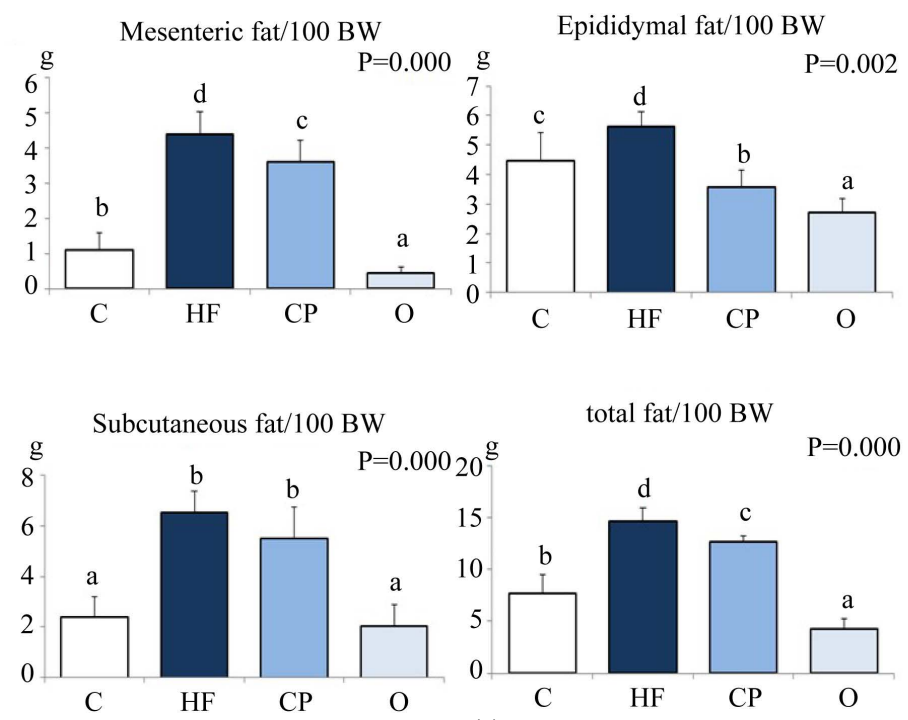
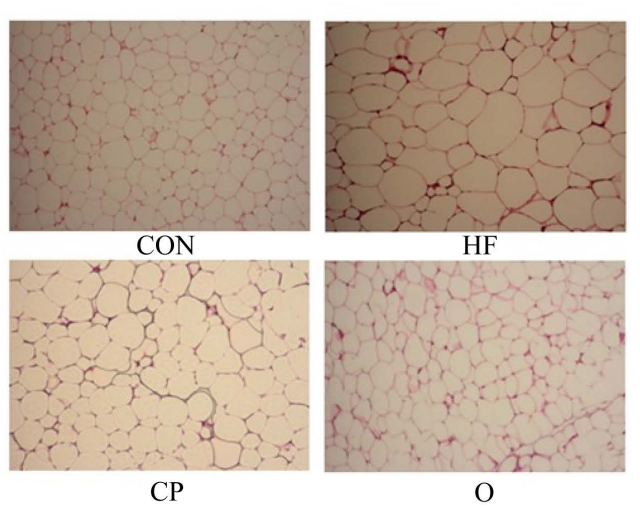

(b)

Figure 2. Effect of citrus peel treatment on adipose tissue weight (a) and morphology (b). High fat diet increased the weight in all fat areas (epididymal, mesenteric, subcutaneous) and citrus peel supplement blocked lipid accumulation Histological detection of H\&E stained epididymal adipose tissue under light microscope at magnification of $\times 100$. C: Chow diet, HF: High fat diet, CP: High fat diet + Citrus peel $(300 \mathrm{mg} / \mathrm{kg})$, O: High fat diet + Orlistat (30 mg/kg). Mean \pm SE (n = 6 for each group). a-d mean with different letters are statistically significantly different $(\mathrm{p}<0.05)$ by ANOVA and Duncan's multiple range test. 


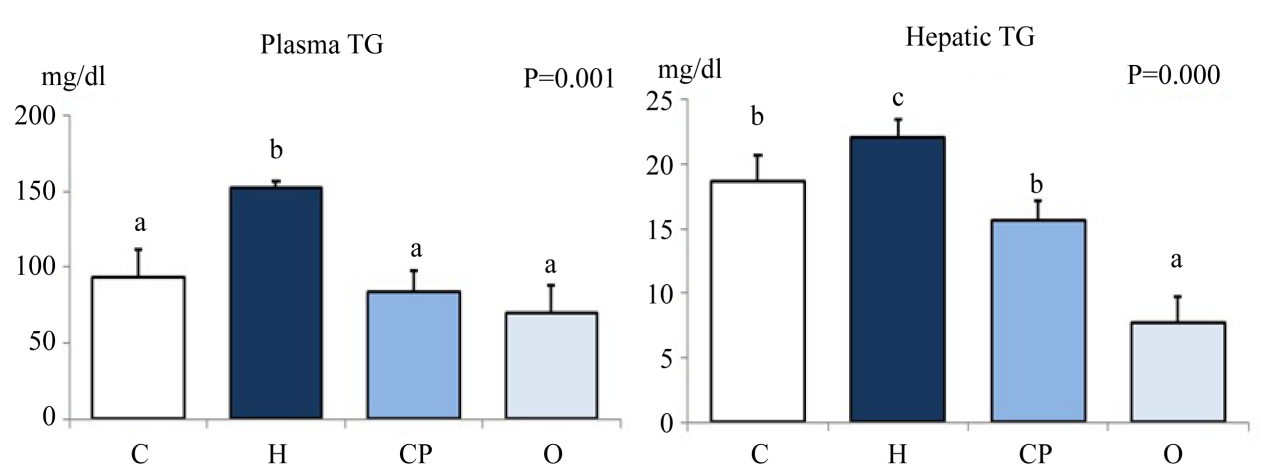

(a)
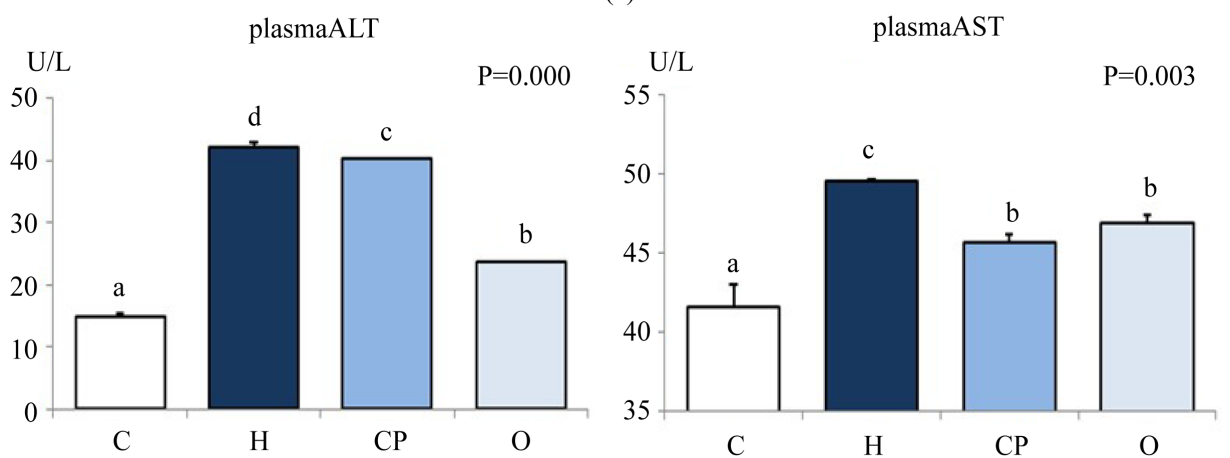

(b)

\begin{abstract}
Figure 3. Effects of diet on plasma and hepatic TG and plasma ALT and AST levels. High fat treatment increased TG in both blood plasma and hepatic tissue, but citrus peel treatment blocked the high fat effect (a). Citrus peel blocked increases in AST and ALT levels in blood plasma caused by high fat diet (b). In the groups, C: Chow diet, HF: High fat diet, CP: High fat diet + Citrus peel (300 $\mathrm{mg} / \mathrm{kg}$ ), O: High fat diet + Orlistat (30 mg/kg). Mean \pm SE ( $=6$ for each group). a-d means with the different letters are statistically significantly different $(\mathrm{p}<0.05)$ by ANOVA and Duncan's multiple range test.
\end{abstract}

(Figure 2), it was concluded that there was no statistically significant difference in adipocyte size between the C, $\mathrm{CP}$, and $\mathrm{O}$ groups; however, the adipocyte size of the HF group was noticeably bigger than that of the other groups at $41.89 \pm 1.44 \mu \mathrm{m} . \mathrm{CP}$ and orlistat treatment had similar effects on adipocyte size with results of $23.47 \pm$ $1.84 \mu \mathrm{m}$ and $21.22 \pm 0.72 \mu \mathrm{m}$, respectively.

\title{
3.5. Lipid Metabolism and Inflammation
}

Expression levels of AMPK mRNA were down-regulated in the HF group, but they were elevated with CP treatment. On the other hand, expression levels of C/EBP- $\alpha$ were up-regulated by $25.59 \%$ under the inducement of a high fat diet. There were no significant differences among the other groups. Similar effects were observed for the expression levels of ACC mRNA with up-regulation induced by a high fat diet (42.29\%). Expression levels of P-AMPK/AMPK, pHSL-/HSL, and FAS proteins were elevated notably by a high fat diet. However, the CP group showed further enhancement of down-regulation of FAS expression, while the $\mathrm{O}$ group showed a similar enhanced down-regulation towards the expression of pHSL-/HSL. In contrast, P-AMPK/AMPK expression was up-regulated by both the CP (30.79\%) and O (54.67\%) groups and there were decreased expression levels with a high fat diet (Figure 4). Although IL-6 expression was upregulated by $123.27 \%$ in the O group and by $158.14 \%$ in the HF group, TNF- $\alpha$ expression decreased by $53.09 \%$ in the CP group and was elevated by $284.22 \%$ in the HF group and $225.52 \%$ in the O group. However, there was no significant difference between the HF and $\mathrm{C}$ groups in protein levels of these factors. In addition, in comparison with the HF group, CP and orlistat treatment down-regulated TNF- $\alpha$ expression. Furthermore, there was no significant effect of MCP-1 regulation on protein level from a high fat diet; however, it was down-regulated by CP and orlistat treatment. 


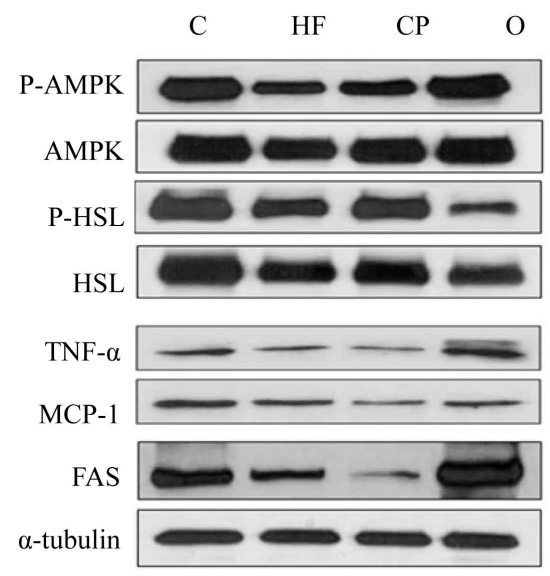

(a)

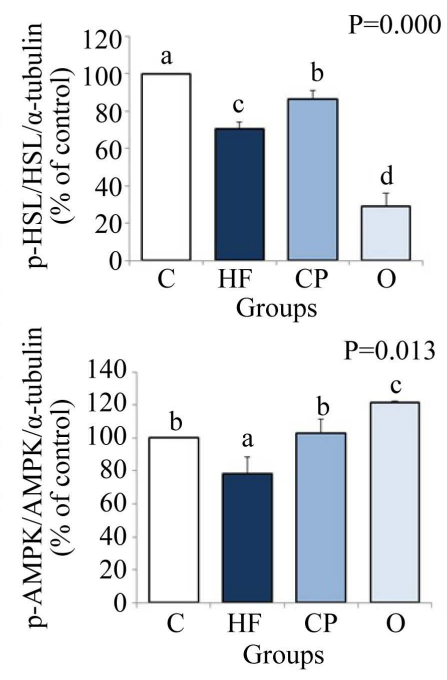

(b)

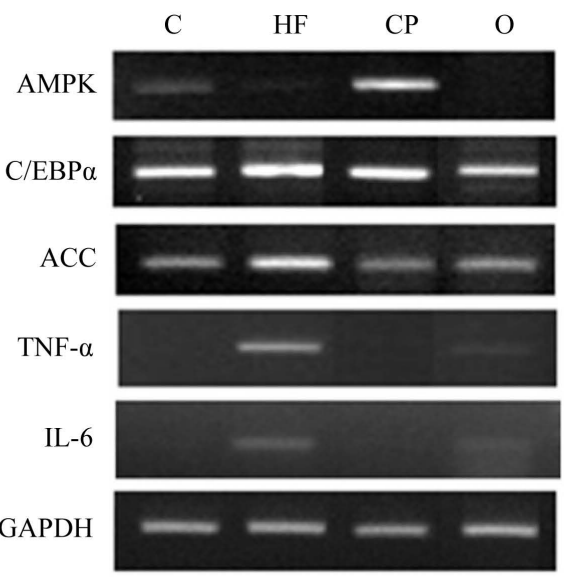

(c)

Figure 4. Regulative effect of citrus peel in adipogenesis and inflammation responsive protein and mRNA levels. After 12 week treatment, TNF- $\alpha$, MCP-1, and FAS were down-regulated while AMPK, p-AMPK, HSL, and p-HSL were up-regulated for protein level (a). Also p-AMPK/AMPK and p-HSL/HSL ratios were increased by citrus peel extract (b). AMPK was up-regulated while ACC, IL-6, TNF- $\alpha$, and C/EBP- $\alpha$ were down-regulated by citrus peel extract (c). GAPDH was used as an internal standard for RT-PCR and $\alpha$-tubulin was used as an internal standard for Western blot. C: Chow diet, HF: High fat diet, CP: High fat diet + Citrus peel (300 mg/kg), O: High fat diet + Orlistat (30 mg/kg).

\section{Discussion}

This study compared CP treatment with an untreated HF group and orlistat treatment. Our results show that the $\mathrm{CP}$ treatment is comparable with that of orlistat treatment, is not as good as orlistat's effects on the reduction of fat accumulation, and provide sufficient credibility that $\mathrm{CP}$ is a natural source for new anti-obesity candidate. Flavonoids are aromatic secondary plant metabolites that are important because of their nutraceutical value. They show several bioactivities such as anti-adipogenic, antiviral, antimicrobial, and anti-inflammatory activities [16]-[20]. Citrus fruit is an important source for bioactive flavonoids, which have been targeted by many studies searching for natural health-promoting substances that can act on different disorders such as adiposity and obesity [21] [22]. Several clinical trials have demonstrated that citrus flavonoids have positive effects against obesity such as lowering of LDL-c and TG and have a negative effect on pro-inflammatory cytokines by regulation on hepatic enzyme activities [23]-[25].

The most abundant flavonoids in citrus fruit are hesperidin, narirutin, and neohesperidin; and they are mainly extracted by using aqueous-based ethanol or methanol solutions [26]. In most species, the amount of hesperidin in citrus fruit peel is recorded as being higher than that of narirutin [27], but a previous study showed that hesperidin has a slightly higher solubility in methanol and water than narirutin [28]. Considering that the extraction from C. unshiu peel was conducted in $70 \%$ ethanol, the narirutin content was expected to be higher than that of hesperidin.

In this study, we compare the effect of CP with that of orlistat because orlistat is a pancreatic lipase inhibitor that affects absorption of fat in the intestine and has been marketed for over ten years as a commercial drug designed for treatment of obesity [29]. It works by inhibiting gastric and pancreatic lipases, the enzymes that break down TG in the intestine. When lipase activity is blocked, triglycerides from the diet are not hydrolyzed into absorbable free fatty acids and instead are excreted undigested. Failure to digest TG gradually decreases the TG level in blood plasma. Ding et al. [30] proved that CP extract blocked the increasing effect of high fat diet on TG and TC in hepatic cells. Similarly in this study, even though CP did not lower TG and TC levels to the same extent as with orlistat, it did arrest the elevation of TG and TC levels induced by high fat diet in both blood plasma and hepatic tissue so that they were at the same level as the control group. In addition, after 12 weeks of treatment, orlistat decreased the body weight and food efficiency of mice with high fat-induced obesity, while $\mathrm{CP}$ extract also decreased food efficiency and had a positive effect on blocking body weight gain. Previous re- 
search showed that citrus fruit extracts with various ethanol concentrations had a negative effect on body weight gain [30] [31]. Regulation of AST and ALT levels in plasma is associated with hepatic toxicity [32]. Current data demonstrates that high fat diet feeding for 22 weeks causes a significant increase in blood plasma AST and ALT levels compared with a control group. Our study showed that hepatic toxicity was not induced after treatment with CP even with an increased concentration up to $1000 \mathrm{mg} / \mathrm{kg}$, and also there was decreased pressure on hepatic tissue that was promoted by high fat diet.

It is known that there is a correlation between lipoprotein cholesterol changes and obesity. In obesity cases there is often a decrease in HDL-c level, and elevation of HDL-c level is one the targets for anti-obesity treatment [33]. Besides, HDL is involved with activating a specific signaling pathway that down-regulates expression of inflammatory cytokines such as TNF- $\alpha$ [34] [35]. Our results show that CP treatment notably increases HDL-c level and, accordingly, it significantly decreases LDL-c level. Also the results show that CP is more effective than orlistat for elevation of HDL-c.

In most cases, a comparison with the HF group shows that the CP group mitigated the effects of a high fat diet; however, the $\mathrm{O}$ group regulated strongly changes in adipose tissue by causing changes that were either more or less than the levels of the $\mathrm{C}$ group, whereas regulation by the $\mathrm{CP}$ group caused only changes that produced levels similar to those in the $\mathrm{C}$ group. This difference was also observed with measurement of epididymal, mesenteric, and subcutaneous fat weight. In addition, H\&E staining of adipose tissue revealed the same effective changes of lipid accumulation in adipose tissue. Considering these results, it is suggested that citrus peel treatment is effective in a different way to orlistat treatment.

In order to underlie and provide insights on the action mechanism of CP, Western blotting and RT-PCR experiments were conducted. High-fat diet lowered the p-AMPK/AMPK ratio compared with normal diet, while treatment with CP extract and orlistat produced a notable increase in the p-AMPK/AMPK ratio compared with the $\mathrm{C}$ group. A lowering of TG and TC content and elevated p-AMPK levels indicates that both the CP and O groups had a trend towards elevated cellular energy metabolism, which might cause the anti-obesity effect and also relieve the high fat-induced hepatic stress.

A different action mechanism was shown by protein levels of p-HSL and FAS, where the CP and O groups exerted opposite effects on the p-HSL/HSL ratio. FAS and HSL are two different key proteins that regulate triglyceride and fatty acid balance. While FAS enzyme contributes to the synthesis of fatty acids, HSL enzyme initiates the catalysis of triglyceride in adipose tissue [36]. As expected, a high fat diet caused HSL activity to be decreased as a result of body weight gain and energy storage. In this context, orlistat is known to exert its effect against obesity and obesity-induced inflammation through blocking of fat absorption; hence, the lowered free fatty acid levels and fat storage. However, unlike orlistat treatment, CP treatment only led to a recovery in the lowered activated HSL levels that brought them closer to those of the normal diet fed C group. Sekiya et al. proved that an absence of HSL inhibits adipogenesis [37]. The distinctive differences between the two groups for HSL-related regulation of obesity suggests that the CP treatment intervened by having an effect on cellular energy metabolism rather than on fat absorption, which was reported also in earlier studies [38]. Coupled with the AMPK protein levels, these results indicate that the effect of CP treatment on cellular fat storage is related to inducing lipid lipolysis and possibly elevating energy metabolism; while orlistat blocks fat absorption into cells, which prevents fat storage and induces energy metabolism. Also FAS protein and mRNA expression decreased with CP treatment, and this result is in agreement with the finding of Ding et al. [30] FAS has a specific function in related energy metabolism, and it is know that there is over expression of FAS under inflammatory circumstances [39]. Knowing that orlistat prevents body weight gain and fat storage and hence adiposity, it was expected that obesity-induced inflammatory cytokines would be affected accordingly. With the suggestion that CP induced lipolysis and lowered blood TG levels, it was also considered that CP affects the pro-inflammatory response; and it is speculated that the increased lipolysis is linked with a stimulated inflammatory response in adipose tissue. Our results suggest that CP treatment relieved hepatic stress induced by adiposity and had the effect of increasing activated HSL, thereby inducing lipolysis.

Meanwhile, the RT-PCR results indicate that CP treatment was able to lower the hepatic inflammatory response that was shown by the increase in mRNA levels of pro-inflammatory factors induced by the HF diet. Adipose tissue lipolysis is known to activate IL-6 production [40]. Consequently, elevation in IL-6 expression was also expected with CP treatment because CP treatment induced lipolysis; however, the CP group had lowered IL-6 production at the gene level while showing anti-inflammatory relief to hepatic stress compared with the HF group. As expected, orlistat was also able to show a slight lowering effect on inflammatory response 
factors, but this was not as strong as with the CP treatment. Pantsulaia et al. suggested that citrus peel extract significantly prevents development of injury by causing a decrease of TNF- $\alpha$ level [41]. Our results show that IL-6 and TNF- $\alpha$ mRNA levels were decreased by CP treatment. Parallel with the suggestion that $C$. unshiu extract intervenes in the cellular energy mechanism and lipolysis, there was notable regulation of C/EBP- $\alpha$ and AMPK mRNA levels by the CP treatment. AMPK is known to play a major role in glucose and lipid metabolism and controls metabolic disorders such as obesity and adipogenesis [42]. On the other hand C/EBP- $\alpha$ encoded protein has the ability to bind promoters and plays an important role in adipogenesis [43]. Lowered C/EBP- $\alpha$ production suggests limited adiposity through adipogenesis, while significantly high AMPK levels suggest elevated fatty acid oxidation and possible inhibition of lipogenesis. Considering that AMPK stimulation also inhibits adipocyte-related lipolysis and lipogenesis, inducement of HSL-C induced lipolysis by the $C$. unshiu extract treatment is likely to be caused by different bioactive molecules in the extract that intervene in a different cellular mechanism in order to show an anti-obesity effect in high fat diet induced obesity mice.

\section{Conclusion}

CP treatment had a similar effect on high fat diet induced obesity as orlistat treatment. CP (300 mg/kg) and orlistat (30 mg/kg) decreased fat accumulation in adipose tissues with reduction of plasma and hepatic TG levels without any hepatic toxicity. CP treatment was more effective than orlistat treatment in causing changes in lipid profiles such as elevation of HDL-c and accordingly a decrease of TC and LDL-c. Although both the CP and O groups showed similar results on fat tissue, $\mathrm{CP}$ was more effective at inhibiting anti-inflammatory cytokines than orlistat. CP regulates adipogenesis, energy metabolism, and lipolysis through the TNF-alpha signaling dependent AMPK pathway. Considering that CP contains different kinds of bioactive substances, mainly flavonoids, these are credited with the reported effects of intervention in different steps of fat metabolism and lipidemiarelated inflammatory response. Although presence of a CP extract was able to regulate high fat diet-induced obesity and relieve the inflammatory response caused by adipocyte-related factors, further studies are needed that focus on the discovery and elucidation of the biochemically responsible chemical constituents for the health beneficial effects in order to understand the underlying action mechanism. Regardless, CP treatment was shown to be effective and to hold a great deal of potential as a treatment against obesity and related inflammation and to be a natural alternative for the safe regulation of the harmful effects caused by high fat diet.

\section{Acknowledgements}

This work is supported by Cooperative Research Program for Agriculture Science \& Technology Development (PJ907089) and Technology Commercialization Support Program, Ministry for Food, Agriculture, Forestry and Fisheries (811003031SU000), Republic of Korea.

\section{Highlights}

- Citrus unshiu peel ethanol extract blocks lipid accumulation.

- Citrus peel ethanol extract decreases TNF- $\alpha$ expressions in obesity induced mice.

- Citrus peel is a promising candidate for developing a natural anti-obesity medicine.

\section{Funds}

This work is supported by Cooperative Research Program for Agriculture Science \& Technology Development (PJ907089) and Technology Commercialization Support Program, Ministry for Food, Agriculture, Forestry and Fisheries (811003031SU000).

\section{References}

[1] Dandona, P., Aljada, A. and Bandyopadhyay, A. (2004) Inflammation: The Link between Insulin Resistance, Obesity and Diabetes. Trends in Immunology, 25, 4-7. http://dx.doi.org/10.1016/j.it.2003.10.013

[2] Jung, U. and Choi, M.S. (2014) Obesity and Its Metabolic Complications: The Role of Adipokines and the Relationship between Obesity, Inflammation, Insulin Resistance, Dyslipidemia and Nonalcoholic Fatty Liver Disease. International Journal of Molecular Sciences, 15, 6184-6123. http://dx.doi.org/10.3390/ijms15046184

[3] Noriyuki, O., Parker, J.L., Lugus, J.J. and Walsh, K. (2011) Adipokines in Inflammation and Metabolic Disease. Na- 
ture Reviews Immunology, 11, 85-97. http://dx.doi.org/10.1038/nri2921

[4] Trayhurn, P. and Beattie, J.H. (2001) Physiological Role of Adipose Tissue: White Adipose Tissue as an Endocrine and Secretory Organ. Proceedings of the Nutrition Society, 60, 329-339. http://dx.doi.org/10.1079/PNS200194

[5] Wellen, K.E. and Hotamisligil, G.S. (2003) Obesity-Induced Inflammatory Changes in Adipose Tissue. Journal of Clinical Investigation, 112, 1785-1788. http://dx.doi.org/10.1172/JCI20514

[6] Gaidhu, M.P. and Ceddia, R.B. (2011) The Role of Adenosine Monophosphate Kinase in Remodeling White Adipose Tissue Metabolism. Exercise and Sport Sciences Reviews, 39, 102-108. http://dx.doi.org/10.1097/JES.0b013e31820ac03e

[7] Cheigh, C.I., Chung, E.Y. and Chung, M.S. (2012) Enhanced Extraction of Flavanones Hesperidin and Narirutin from C. unshiu Peel Using Subcritical Water. Journal of Food Engineering, 110, 472-477. http://dx.doi.org/10.1016/j.jfoodeng.2011.12.019

[8] Balasunram, N., Sundram, K. and Samman, S. (2006) Phenolic Compounds in Plants and Agri-Industrial By-Products: Antioxidant Activity, Occurrence, and Potential Uses. Food Chemistry, 99, 191-203.

http://dx.doi.org/10.1016/j.foodchem.2005.07.042

[9] Ghasemi, K., Ghasemi, Y. and Ebrahimzadeh, M.A. (2009) Antioxidant Activity, Phenol and Flavonoid Contents of 13 Citrus Species Peels and Tissues. Pakistan Journal of Pharmaceutical Sciences, 22, 277-281.

[10] Benavente-Garcia, O. and Castillo, J. (2008) Update on Uses and Properties of Citrus Flavonoids: New Findings in Anticancer, Cardiovascular, and Anti-Inflammatory Activity. Journal of Agricultural and Food Chemistry, 15, 61856205. http://dx.doi.org/10.1021/jf8006568

[11] Oh, Y.C., Cho, W.K., Jeong, Y.H., Im, G.Y., Yang, M.C., Hwang, Y.H., et al. (2012) Anti-Inflammatory Effect of Citrus unshiu Peel in LPS-Stimulated RAW 264.7 Macrophage Cells. The American Journal of Chinese Medicine, 40, 611-629. http://dx.doi.org/10.1142/S0192415X12500462

[12] Min, K.Y., Lee, K.A., Kim, H.J., Kim, K.T., Chung, M.S., Chang, P.S., et al. (2014) Antioxidative and Anti-Inflammatory Activities of Citrus unshiu Peel Extracts Using a Combined Process of Subcritical Water Extraction and Acid Hydrolysis. Food Science and Biotechnology, 23, 1441-1446. http://dx.doi.org/10.1007/s10068-014-0197-y

[13] Jung, H.K., Jeong, Y.S., Park, C.D., Park, C.H. and Hong, J.H. (2011) Inhibitory Effect of Citrus Peel Extract on Lipid Accumulation of 3T3-L1 Adipocytes. Journal of the Korean Society for Applied Biological Chemistry, 54, 169-176. http://dx.doi.org/10.3839/jksabc.2011.028

[14] Folch, J., Lees, M. and Stanley, G.H.S. (1957) A Simple Method for the Isolation and Purification of Total Lipids from Animal Tissues. Journal of Biological Chemistry, 226, 497-509.

[15] Johnson, R., McNutt, P., MacMahon, S. and Robson, R. (1997) Use of the Friedewald Formula to Estimate LDLCholesterol in Patients with Chronic Renal Failure on Dialysis. Clinical Chemistry, 43, 2183-2184.

[16] Yi, Z.B., Yu, Y., Liang, Y.Z. and Zeng, B. (2008) In Vitro Antioxidant and Antimicrobial Activities of the Extract of Pericarpium Citri Reticulatae of a New Citrus Cultivar and Its Main Flavonoids. LWT_Food Science and Technology, 41, 597-603. http://dx.doi.org/10.1016/j.lwt.2007.04.008

[17] Asres, K., Seyoum, A., Veeresham, C., Bucar, F. and Gibbons, S. (2005) Naturally Derived Anti-HIV Agents. Phytotherapy Research, 19, 557-581. http://dx.doi.org/10.1002/ptr.1629

[18] Cushnie, P.T. and Lamb, A.J. (2005) Antimicrobial Activity of Flavonoids. International Journal of Antimicrobial Agents, 26, 343-356. http://dx.doi.org/10.1016/j.ijantimicag.2005.09.002

[19] Kim, H.P., Son, K.H., Chang, H.W. and Kang, S.S. (2004) Anti-Inflammatory Plant Flavonoids and Cellular Action Mechanisms. Journal of Pharmacological Sciences, 96, 229-245. http://dx.doi.org/10.1254/jphs.CRJ04003X

[20] Karadeniz, F., Kim, J.A., Ahn, B.N., Kwon, M.S. and Kong, C.S. (2014) Effect of Salicornia herbacea on Osteoblastogenesis and Adipogenesis in Vitro. Marine Drugs, 12, 5132-5147. http://dx.doi.org/10.3390/md12105132

[21] Lu, Y., Xi, W., Ding, X., Fan, S., Zhang, Y., Jiang, D., et al. (2013) Citrange Fruit Extracts Alleviate Obesity-Associated Metabolic Disorder in High-Fat Diet-Induced Obese C57BL/6 Mouse. International Journal of Molecular Sciences, 14, 23736-23750. http://dx.doi.org/10.3390/ijms141223736

[22] Fukuchi, Y., Hiramitsu, M., Okada, M., Hayashi, S., Nabeno, Y., Osawa, T., et al. (2008) Polyphenols Suppress Diet-Induced Obesity by Up-Regulation of mRNA Levels of the Enzymes Involved in $\beta$-Oxidation in Mouse White Adipose Tissue. Journal of Clinical Biochemistry and Nutrition, 43, 201-209. http://dx.doi.org/10.3164/jcbn.2008066

[23] Bent, S., Padula, A. and Neuhaus, J. (2004) Safety and Efficacy of Citrus aurantium for Weight Loss. American Journal of Cardiology, 15, 1359-1361. http://dx.doi.org/10.1016/j.amjcard.2004.07.137

[24] Haaz, S., Fontaine, K.R., Cutter, G., Limdi, N., Perumean-Chaney, S. and Allison, D.B. (2006) Citrus aurantium and Synephrine Alkaloids in the Treatment of Overweight and Obesity: An Update. Obesity Reviews, 7, 79-88. http://dx.doi.org/10.1111/j.1467-789X.2006.00195.x 
[25] Kelleya, D.S., Adkinsa, Y.C., Zuninoa, S.J., Woodhousea, L.R., Bonnela, E.L., Breksa, A.P., et al. (2015) Citrus Limonin Glucoside Supplementation Decreased Biomarkers of Liver Disease and Inflammation in Overweight Human Adults. Journal of Functional Foods, 12, 271-281. http://dx.doi.org/10.1016/j.jff.2014.11.026

[26] Ma, Y., Ye, X., Fang, Z., Chen, J., Xu, G. and Liu, D. (2008) Phenolic Compounds and Antioxidant Activity of Extracts from Ultrasonic Treatment of Satsuma Mandarin (Citrus unshiu Marc.) Peels. Journal of Agricultural and Food Chemistry, 56, 5682-5690. http://dx.doi.org/10.1021/jf072474o

[27] Gattuso, G., Barreca, D., Garguilli, C., Leuzzi, U. and Coristi, C. (2007) Flavonoid Composition of Citrus Juice. Molecules, 12, 1641-1673. http://dx.doi.org/10.3390/12081641

[28] Ma, Y., Ye, X., Hao, Y., Xu, G., Xu, G. and Liu, D. (2008) Ultrasound-Assisted Extraction of Hesperidin from Penggan (Citrus reticulata) Peel. Ultrasonics Sonochemistry, 15, 227-232. http://dx.doi.org/10.1016/j.ultsonch.2007.03.006

[29] Svendsen, M. and Tonstad, S. (2011) Orlistat after Initial Dietary/Behavioural Treatment: Changes in Body Weight and Dietary Maintenance in Subjects with Sleep Related Breathing Disorders. Nutrition Journal, 10, 21. http://dx.doi.org/10.1186/1475-2891-10-21

[30] Ding, X., Fan, S., Lu, Y., Zhang, Y., Gu, M., Zhang, L., et al. (2012) Citrus ichangensis Peel Extract Exhibits AntiMetabolic Disorder Effects by the Inhibition of PPAR $\gamma$ and LXR Signaling in High-Fat Diet-Induced C57BL/6 Mouse. Evidence-Based Complementary and Alternative Medicine, 2012, Article ID: 678592. http://dx.doi.org/10.1155/2012/678592

[31] Salter, A.M., Fong, B.S., Jimenez, J., Rotstein, L. and Angel, A. (1987) Regional Variation in High-Density Lipoprotein Binding to Human Adipocyte Plasma Membranes of Massively Obese Subjects. European Journal of Clinical Investigation, 17, 16-22. http://dx.doi.org/10.1111/j.1365-2362.1987.tb01220.x

[32] Park, H.Y., Park, Y., Lee, Y., Noh, S.K., Sung, E.G. and Chou, I. (2012) Effect of Oral Administration of Water-Soluble Extract from Citrus Peel (Citrus unshiu) on Suppressing Alcohol-Induced Fatty Liver in Rats. Food Chemistry, 130, 598-604. http://dx.doi.org/10.1016/j.foodchem.2011.07.081

[33] Vozarova, B., Stefan, N., Lindsay, R.S., Saremi, A., Pratley, R.E., Bogardus, C., et al. (2002) High Alanine Aminotransferase Is Associated with Decreased Hepatic Insulin Sensitivity and Predicts the Development of Type 2 Diabetes. Diabetes, 51, 1889-1895. http://dx.doi.org/10.2337/diabetes.51.6.1889

[34] Wang, S. and Peng, D. (2012) Regulation of Adipocyte Autophagy-The Potential Anti-Obesity Mechanism of High Density Lipoprotein and Apolipoprotein A-I. Lipids in Health and Disease, 11, 131. http://dx.doi.org/10.1186/1476-511X-11-131

[35] Guha, M. and Mackman, N. (2002) The Phosphatidylinositol 3-Kinase-Akt Pathway Limits Lipopolysaccharide Activation of Signaling Pathways and Expression of Inflammatory Mediators in Human Monocytic Cells. The Journal of Biological Chemistry, 277, 32124-32132. http://dx.doi.org/10.1074/jbc.M203298200

[36] Xu, X., Wei, X., Yang, Y., Niu, W., Kou, Q., Wang, X., et al. (2015) PPAR $\gamma$, FAS, HSL mRNA and Protein Expression during Tan Sheep Fat-Tail Development. Electronic Journal of Biotechnology, 18, 122-127. http://dx.doi.org/10.1016/j.ejbt.2015.01.004

[37] Sekiya, M., Osuga, J.I., Okazaki, H., Yahagi, N., Harada, K., Shen, W.J., et al. (2004) Absence of Hormone-Sensitive Lipase Inhibits Obesity and Adipogenesis in Lep ${ }^{\text {ob/ob }}$ Mice. The Journal of Biological Chemistry, 279, 15084-15090. http://dx.doi.org/10.1074/jbc.M310985200

[38] Zechner, R., Kienesberger, P.C., Haemmerle, G., Zimmermann, R. and Lass, A. (2009) Adipose Triglyceride Lipase and the Lipolytic Catabolism of Cellular Fat Stores. Journal of Lipid Research, 50, 3-21. http://dx.doi.org/10.1194/jlr.R800031-JLR200

[39] Consolazio, A., Alò, P.L., Rivera, M., Iacopini, F., Paoluzi, O.A., Crispino, P., et al. (2006) Overexpression of Fatty Acid Synthase in Ulcerative Colitis. American Journal of Clinical Pathology, 126, 113-118. http://dx.doi.org/10.1309/PUBVQNDNVQKJVC8M

[40] Ji, C., Chen, X., Gao, C., Jiao, L., Wang, J., Xu, G., et al. (2011) IL-6 Induces Lipolysis and Mitochondrial Dysfunction, but Does Not Affect Insulin-Mediated Glucose Transport in 3T3-L1 Adipocytes. Journal of Bioenergetics and Biomembranes, 43, 367-375. http://dx.doi.org/10.1007/s10863-011-9361-8

[41] Pantsulaia, I., Iobadze, M., Pantsulaia, N. and Chikovani, T. (2014) The Effect of Citrus Peel Extracts on Cytokines Levels and T Regulatory Cells in Acute Liver Injury. BioMed Research International, 2014, Article ID: 127879. http://dx.doi.org/10.1155/2014/127879

[42] Carling, D. (2004) The AMP-Activated Protein Kinase Cascade-A Unifying System for Energy Control. Trends in Biochemical Sciences, 29, 18-24. http://dx.doi.org/10.1016/j.tibs.2003.11.005

[43] Park, T. and Kim, Y. (2011) Phytochemicals as Potential Agents for Prevention and Treatment of Obesity and Metabolic Diseases. Anti-Obesity Drug Discovery and Development, 1, 1-48. 